\title{
INSCRIPCIONES ROMANAS DE PATERNA, VALENCIA Y RIBA-ROJA DE TÚRIa (TerRItorium de Valentia, Hispania Citerior)
}

Presentamos en estas páginas once nuevas inscripciones romanas sobre diversos soportes, que han aparecido en la última década en diferentes excavaciones en localidades próximas a Valencia y en la propia ciudad, todas ellas dentro del territorium de la antigua Valentia.

Palabras clave: inscripciones romanas, bronce, estela, instrumentum domesticum, ponderales.

\section{Roman inscriptions from Paterna, Valencia, and Riba-roja de Túria (Territorium of Valentia, Hispania Citerior)}

This article presents eleven new Roman inscriptions on various supports that were found in the last decade in different excavations in locations situated in the vicinity of Valencia and in the city itself, all of them within the territorium of the ancient city of Valentia.

Key words: Roman inscriptions, bronze, funerary monuments, instrumentum domesticum, pounds.

\section{PATERNA. VILLA SUBURBANA RÍO 3}

Los trabajos de excavación llevados a cabo entre los años 2007 y 2011 en la denominada villa romana suburbana Río 3 de Paterna (Valencia) pusieron al descubierto un numeroso conjunto de materiales arqueológicos romanos de todo tipo, entre los que se encontraban una serie de soportes epigráficos que se estudian en estas páginas ${ }^{1}$. La villa disponía de una parte residencial y de otra dedicada a la explotación agrícola y a las actividades artesanales. Dado que su ocupación se prolongó desde el s. I a. C. hasta el VII d. C., en el lugar se llevaron a cabo numerosas remodelaciones, especialmente en la etapa romana, y junto a él fue creciendo una necrópolis a la que pertenecerían una parte de los elementos epigráficos que presentamos.
Los nuevos hallazgos incluyen un pequeño fragmento de placa de bronce, que constituye uno de los escasos hallazgos de epígrafes sobre este material en tierras valencianas, una estela funeraria, dos fragmentos de placas de mármol, un fragmento de dolium con inscripción y un interesantísimo vaso de terra sigillata con un grafito que documenta por primera vez el uso del verbo emo,-ere en contextos cerámicos de la península Ibérica. Desafortunadamente, ninguna de estas piezas se encontró en su emplazamiento original, sino que fueron halladas en niveles de amortización, asociadas a estructuras tardías de la villa rústica. Con anterioridad a estos descubrimientos, en el término de Paterna (Valencia) sólo conocíamos un altar funerario romano dedicado por el liberto [E]uphemus a su patrona Teren[ti]a, que 
apareció en la partida de La Presa (La Cañada) en $1976^{2}$. Las nuevas evidencias epigráficas son las que referimos a continuación.

\section{INSCRIPCIÓN FUNERARIA (fig. 1)}

Fragmento de una placa en bronce rota por arriba y por la izquierda, que conserva aún parte de los bordes inferior y derecho del soporte original. Sus dimensiones son $(21,9) \times(19) \times 0,2 \mathrm{~cm}$. En la parte de abajo presenta un recorte de 2,4 x 2,4 cm, a $12 \mathrm{~cm}$ del ángulo inferior derecho, destinado a recibir la grapa metálica que fijaba la placa a una pared o a un monumento. A $1,5 \mathrm{~cm}$ del borde derecho se observa una finísima incisión vertical que recorre el lateral de la pieza y que constituye la huella del proceso de ordinatio. La parte conservada del texto ocupa la parte superior de la placa, en donde se observan restos de tres líneas. Por debajo de ellas hay un espacio libre de $11 \mathrm{~cm}$. El texto está incompleto por la izquierda y sólo presenta restos de tres renglones. La altura de las letras, de arriba a abajo, es de 2 (v. 1), 1,9 (v. 2) y $1,7 \mathrm{~cm}$ (v. 3; la I longa de la tercera línea mide $2,1 \mathrm{~cm})$. Los espacios interlineares miden $1,5,1,5$ y 2 $\mathrm{cm}$. La placa se halló en el nivel de amortización de la

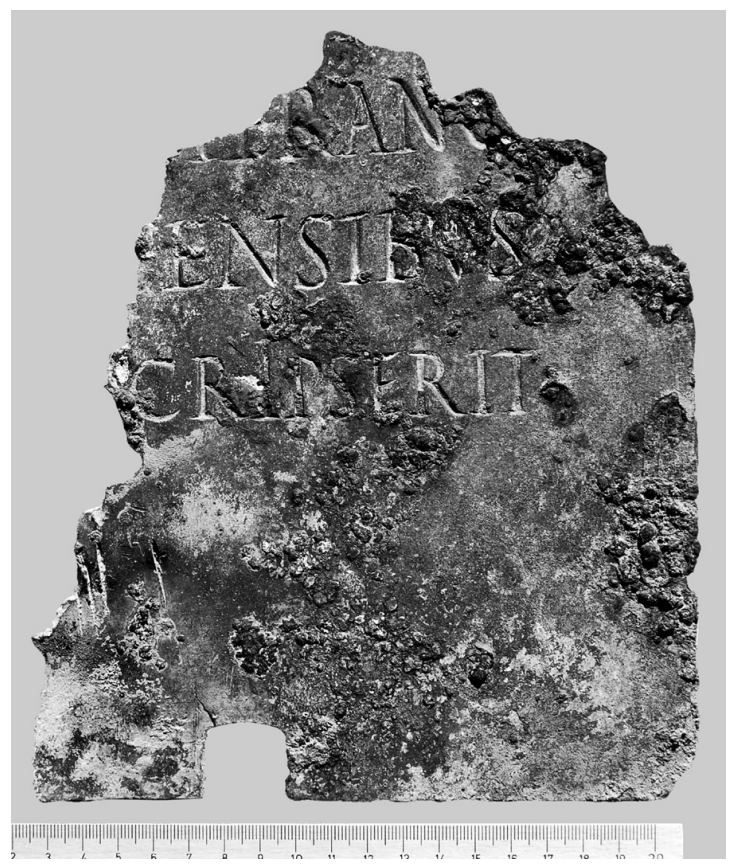

Fig. 1. Inscripción funeraria en bronce procedente de Paterna. boca del praefurnium de los baños de la villa (UE 2226; inv. 3428). Se conserva en el Museo de Cerámica de Paterna, donde pudimos verla en diciembre de 2012 y donde la fotografió R. Cebrián.

\section{$[---]+$ erano \\ $[---]+e n s i b u s$ \\ [---]cripserit \\ $-----?$}

$1+$ es parte inferior de asta vertical, seguramente correspondiente a una $\mathrm{T}$ dado que la letra que sigue es una E, de la que se conserva sólo la mitad inferior. $-2+$ es parte de un trazo vertical de una posible M. -3 en el borde izquierdo los restos de óxido impiden decir si hay restos de una letra antes de la $\mathrm{C}$.

A la vista de las evidencias conservadas, la restitución más probable en el verso inicial es [--- ve]terano. El segundo verso da idea de la enorme laguna perdida por la izquierda, pues lo que se ve es sólo el final de una edad indicada en ablativo en la forma annis + mensibus (+ diebus). Dado que en la primera línea hay un dativo, y en la tercera el verbo se conjuga en tercera persona de singular, ese verso precisa de un sujeto que debía estar emplazado en el último renglón, entre la edad y el verbo, donde habría que esperar la identidad de un miembro de la familia o de un compañero de armas del veteranus citado. Cualquiera de las opciones supone también una considerable longitud de la parte de la placa que ha desaparecido. En la parte inferior debió de existir un cuarto verso, más corto y centrado, que habría perecido con la fractura, en donde debía leerse el final de la inscripción.

El uso de la forma verbal scripserit es muy escaso en la epigrafía romana y se reduce a documentos jurídicos entre los que se incluyen la ley colonial de Urso en la Baetica (Urs. CXXXI), una sentencia sobre límites territoriales en Macedonia en tiempos del emperador Adriano (CIL III 12306 = III 586; Dessau ILS 5947a), un fragmento jurídico de Pompeya (CIL IV 7521), el conocido homenaje de Aquileia a C. Minicius Italus (CIL V 875; Dessau ILS 1374) y, por lo que a nosotros aquí nos interesa, el epígrafe funerario de Roma que M. Popilius Euphemus y Popilia Moschis dedicaron a su hijo M. Popilius Zosimianus con la advertencia de que quisquis hoc monumentum violaverit aut titulum deasciaverit aliove quo nomine inscripserit debía pagar al erario público una multa de 20.000 sestercios: dabit in aerarium p(opuli) R(omani) HS XX m(ilia) n(uтmum) (CIL VI 24799; Dessau ILS 8220). 
Si la inscripción de Paterna incluye, como parece probable, la amenaza de una pena sepulcral para los posibles usuarios posteriores del sepulcro, es decir, de una multa por reutilizar el lugar de enterramiento, no se trataría del primer testimonio descubierto en Hispania, donde ya conocemos un epígrafe jiennense con una referencia expresa al castigo económico previsto por la violatio del locus a favor de la res publica Aiungitanorum ${ }^{3}$.

Es decir, el nuevo texto de Paterna pudo decir, exempli gratia, algo parecido a esto:

$$
\begin{aligned}
& \text { [--- ve]terano } \\
& \text { [-- annis --- ] mensibus } \\
& \text { [-- diebus (?) --- s]cripserit } \\
& \text { [dabit? --- HS --- m(ilia) n(ummum)] }
\end{aligned}
$$

El bronce fue el soporte tradicional de la documentación jurídica pública y privada de la antigüedad por su resistencia a la intemperie y su longevidad frente a soportes más débiles (De Hoz 1999: 433-470; Beltrán Lloris 1999: 21). Las tabulae aeneae constituyen el soporte ordinario de textos de patronatum y hospitium, de rescriptos imperiales, leyes locales, dedicaciones institucionales, tabulae defixionum, documentos de honesta missio, etc., de los que la península Ibérica ha sido pródiga ${ }^{4}$. Algunos de esos documentos de bronce, pese a quedar restringidos en su contenido al ámbito privado ${ }^{5}$, contenían -como parece ser el caso- advertencias de carácter público que entraban en las categorías de las acciones punibles por parte de las instituciones, lo que justificaría el empleo de este tipo de soporte. Aunque escasean, tampoco faltan las inscripciones funerarias en bronce, como en el caso que nos ocupa ${ }^{6}$.

Atendiendo al tipo de letra, la inscripción parece poder fecharse en el s. II d.C.

\section{INSCRIPCIÓN FUNERARIA (figs. 2 y 3)}

Estela funeraria de cabecera semicircular en piedra arenisca local, rota por su parte inferior. La parte superior está muy alterada y la cabecera ha perdido casi toda su forma original. La superficie frontal apenas fue alisada antes de grabar el texto -tampoco la parte posterior de la estela- mientras que las caras laterales están más o menos alisadas. Sus dimensiones son (67) x 39,5 x $27 \mathrm{~cm}$. En la parte inferior conserva un pequeño zócalo de circ. $15 \mathrm{~cm}$ de altura, perteneciente a la parte del monumento que iba clavada en el suelo. En la parte superior de la cara

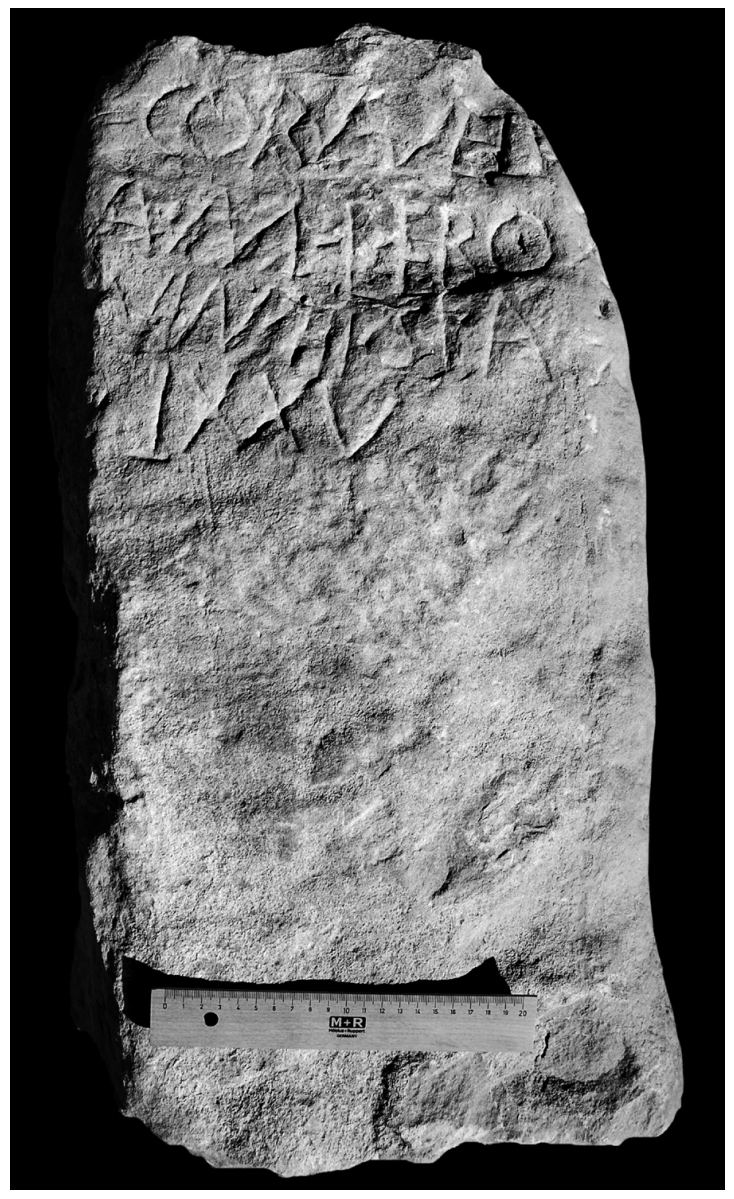

Fig. 2. Estela funeraria de Paterna.

lateral izquierda presenta un orificio de $5 \mathrm{~cm}$ de diámetro, que parece original. El texto está toscamente grabado, con poca profundidad en los trazos y sin cuidar la horizontalidad de las líneas, por lo que la altura de las letras es muy irregular. Su altura es de 4,3 (v. 1), 5,8/5,3 (v. 2), $5 / 4,5$ (v. 3) y $5,4 / 4,5 \mathrm{~cm}$ (v. 4). Los espacios interlineares miden $2,6,1,5$ y $0,6 \mathrm{~cm}$. Presenta interpunciones circulares en la línea segunda y triangulares, aunque muy deformadas, en la tercera. Por encima y debajo del texto hay un espacio libre de 6 y $42 \mathrm{~cm}$ respectivamente. Se descubrió en una de las estancias dedicadas a la producción en la villa, donde había sido reaprovechada en un muro posterior a la fecha de su tallado (UE 5001; inv. 9986). Se conserva en el Museo de Cerámica de Paterna, donde pudimos verla en diciembre de 2012 y donde la fotografió R. Cebrián. 
Corneli-

$a \cdot M($ arci $) \cdot l($ iberta $) \cdot$ Ebo-

$\operatorname{vin}(a) \cdot \operatorname{hi}(c) \cdot \operatorname{si}($ ta $) \cdot a($ nnorum $)$

$L X X V$

Traducción: Aquí yace Cornelia Ebovina, liberta de Marcus (Cornelius), de setenta y cinco años de edad.

La difunta, una liberta de la gens Cornelia, presenta un cognomen completamente desconocido hasta ahora y ni siquiera se pueden aducir formas próximas salvo que prescindamos de la $\mathrm{E}$ inicial $^{7}$, claramente visible en el monumento. El texto dice Ebovina y no Erovina, como podría desprenderse de la fig. 2; la foto de detalle de la fig. 3 muestra que la letra B se cierra claramente por la parte inferior.

Dentro de su tosquedad, el monumento es muy interesante por la presencia del corte asilábico al final del primer renglón, la abreviatura de la voz annorum con una sola vocal ${ }^{8}$ y la curiosa forma de indicar la fórmula funeraria ${ }^{9}$. A este respecto merecen destacarse tanto un epígrafe de Toledo en el que expresamente la fórmula dice hi(c) si(tus) ${ }^{10}$ como especialmente uno de Alarcón (Cuenca), hoy perdido, en el que una vieja fotografía -conservada en el Archivo de la provincia de Castilla de la Compañía de Jesús en Alcalá de Henares (Madrid)- permite leer hi(c) si(ta) est (CIL II 5896) ${ }^{11}$. Hay que reseñar también la forma arcaica de escribir el numeral $L$ en forma de $T$ invertida, con un trazo vertical que corta por el centro otro horizontal más corto. La forma de esta última letra asegura una datación temprana, quizá no más allá del tercer

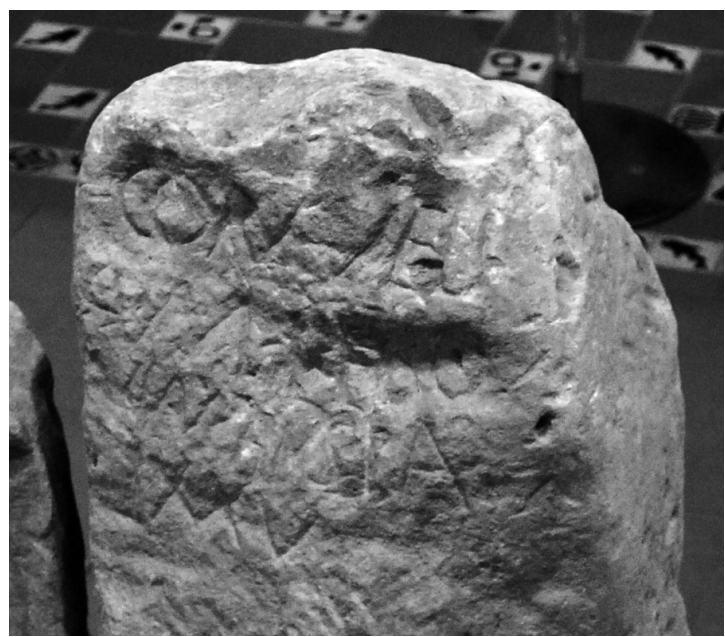

Fig. 3. Detalle de la cabecera en la estela funeraria de Paterna. cuarto del s. I d.C. (Stylow 1995: 222); esta forma del numeral en Hispania se documenta especialmente en la Baetica, en donde conocemos su uso en miliarios y en inscripciones funerarias (cf. por ejemplo $C I L \mathrm{II}^{2} / 7,881$ ).

\section{INSCRIPCIÓN FUNERARIA (fig. 4)}

Fragmento de una placa de mármol blanco con veta gris, rota por arriba y por la izquierda, con todas las caras pulidas. Sus dimensiones son $(11,5)$ x $(18,7)$ x $1,9 \mathrm{~cm}$. La altura de las letras es de 2,5 (v. 1), 2 (v. 2) y 1,5 cm (v. 3), con espacios interlineares que miden $1,2,1,5$ y $0,9 \mathrm{~cm}$ respectivamente. Al final de los renglones 1 y 3 aparecen sendos motivos decorativos en forma dos hederae simplificadas y colocadas una sobre la otra. El fragmento fue descubierto en un nivel de relleno (UE 2008; inv. 3567). Se conserva en el Museo de Cerámica de Paterna, donde lo describió y fotografió R. Cebrián.

En la parte perdida por arriba debieron desaparecer al menos dos o tres líneas, una de ellas dedicada a la invocación a los Dioses Manes y una -o dos- con el nombre de la difunta. Si atendemos a la ordinatio del texto, la placa ha perdido por la parte izquierda sólo unos pocos centímetros, los que hacen falta para el margen izquierdo y la letra $\mathrm{V}$ de [u]xori en el último renglón y para las dos primeras letras de la fórmula funeraria en el primero. Es decir, en el segundo de los renglones conservados debe encontrarse-abreviado con no más de tres letras si atendemos al espacio disponible- el nomen del dedicante, que debería ser, por lo tanto, [Co]r(nelius] y no [Vale]r(ius]. Según eso, el texto debe decir:

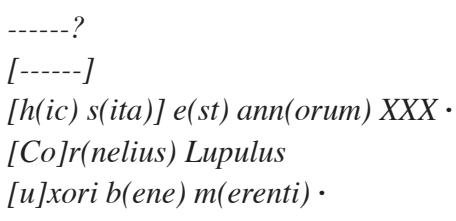

Traducción: Aquí yace [---] de 30 años de edad. Cornelius Lupulus dedicó este monumento a su queridísima esposa.

El nomen del dedicante es el tercero de los más corrientes en Hispania (Abascal 1994: 29) y su presencia no es relevante, aunque hay que recordar -porque el nomen se repite en la inscripción anterior- que los Cornelii se reconocen en muchos epígrafes del $\mathrm{S}$ del conventus Tarraconensis y que al oriente de Valentia se encuentra Edeta, la patria del senador M. Cornelius Nigrinus Curiatius 


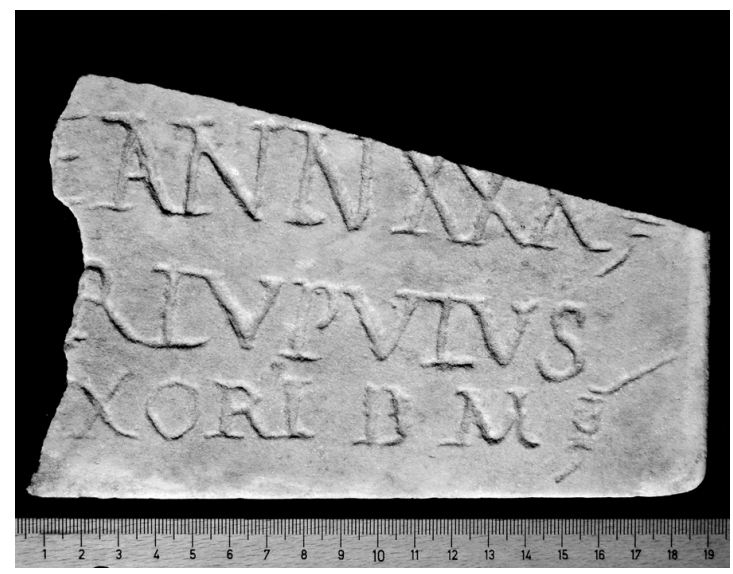

Fig. 4. Placa funeraria de mármol.

Maternus (Alföldy y Halfmann 1973). Lupulus, el cognomen del dedicante (Kajanto 1965,328), se repite en Hispania en tres ocasiones en masculino y cuatro en femenino ${ }^{12}$.

El tipo de letra, combinado con la fórmula funeraria [h(ic) $s($ ita $]$ ] e(st) y la dedicación b(ene) m(erenti), aconseja una datación en la primera mitad del siglo III.

\section{INSCRIPCIÓN FUNERARIA (figs. 5 a 7)}

Fragmento de una placa de mármol blanco con veta gris, rota por arriba y por la derecha, que conserva casi intactos su borde izquierdo e inferior, pulida por todas las caras y cuyas dimensiones son $(16,5)$ x (23) x 3,6 cm. La altura de las letras es de 3,6 (v. 1) y $2,8 \mathrm{~cm}$ (v. 2), separadas por un interlineado de $1,5 \mathrm{~cm}$. El área epigráfica está enmarcada por una cyma recta, tallada a $3 \mathrm{~cm}$ del borde de la cara frontal. La placa presenta también una cyma en la parte posterior, lo que parece indicar un reaprovechamiento de la pieza en época posterior para el placado marmóreo de la pared de una estancia de la villa. Se descubrió en un nivel de relleno (UE 2008; inv. 3568). Se conserva en el Museo de Cerámica de Paterna, donde lo describió y fotografió R. Cebrián.

\section{$----\cdot$ \\ [-- hoc] mo- \\ nime[ntum here] \\ dem [non sequet(ur)]}

La restitución del texto no plantea ninguna dificultad a la vista del paralelo casi exacto de $C I L I^{2} / 7,40$, en donde

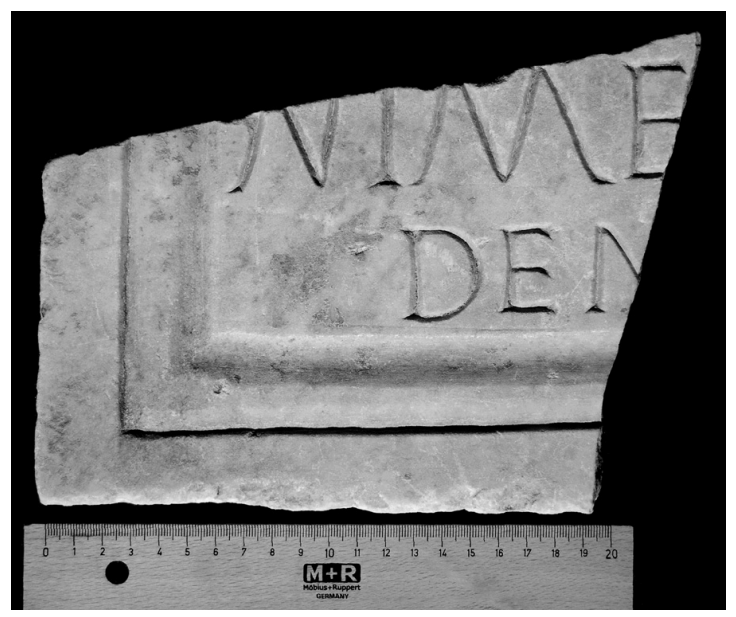

Fig. 5. Anverso de la placa de mármol no 4 .

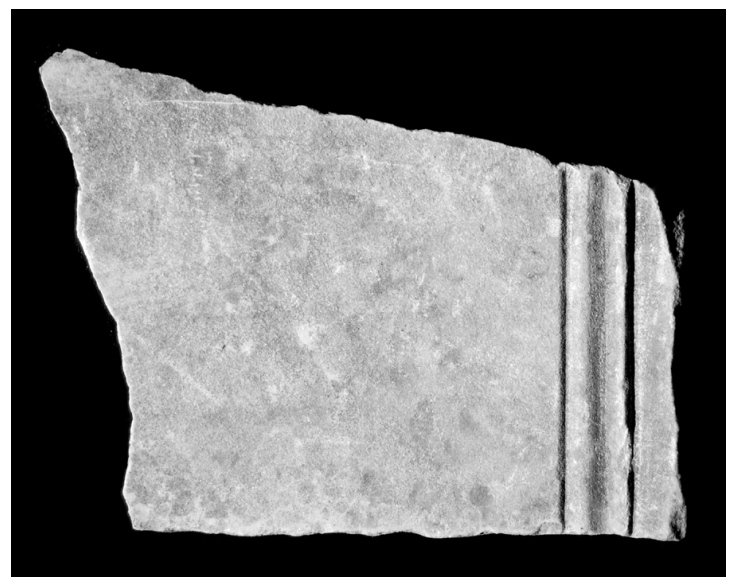

Fig. 6. Reverso de la placa de mármol nº 4 .

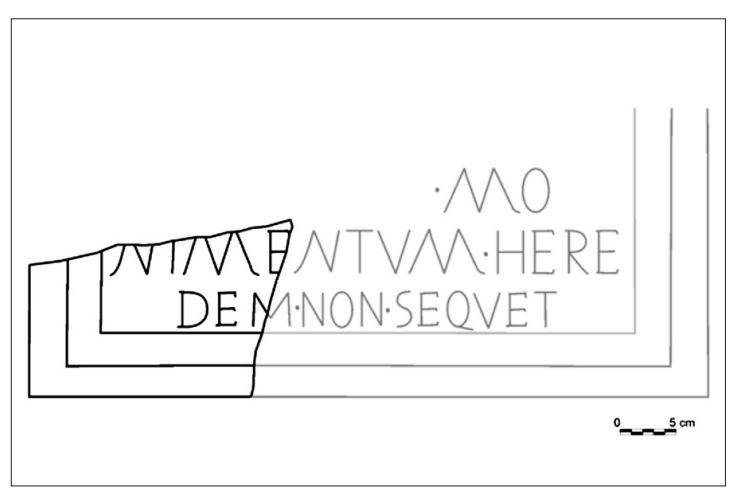

Fig. 7. Restitución probable del texto de la placa ${ }^{\circ} 4$. 
puede leerse hoc monimentum seu sepulcrum est heredem non sequitu[r]. La fórmula simplificada suele aparecer abreviada con las letras H M H N S, y se construye sobre la premisa de que el sepulcro es el monumentum que alberga el cuerpo del difunto o las reliquias (Dig. 11, 7, 42) como ya hiciera notar Stylow en la edición del texto cordobés. La fórmula abreviada estaba ya atestiguada en el área valenciana, donde la encontramos en una inscripción de Albocàsser (Castellón) (CIL II²/14, 764), en dos inscripciones, hoy perdidas, de Edeta (CIL II ${ }^{2} / 4,164$ y 171$)$ y en nueve de Saguntum y su territorio $\left(C I L \mathrm{II}^{2} / 14,358,407,521\right.$, $523,601,645,653,654,741)$. En el área valenciana, sobre todo en Saguntum, hay ejemplos que evidencian el uso de la fórmula funeraria hoc monumentum heredes non sequetur entre matrimonios de libertos, que permite a la mujer el derecho de sepultura en calidad de familiar y no de heredera ${ }^{13}$.

La forma monimentum por monumentum es corriente; sólo Dessau (ILS cf. p. 823) en su reducida selección epigráfica recoge más de veinte testimonios.

La inscripción debería poder fecharse, por el tipo de letra, en la primera mitad del s. II d.C.

\section{INSCRIPCIÓN DE CAPACIDAD (fig. 8)}

Fragmento de pared de dolium. En su pared exterior conserva la parte izquierda de un grafito ante cocción referido a la capacidad del recipiente, como suele ser habitual. Las dimensiones del fragmento son (40) x (35) x 3,4 $\mathrm{cm}$. La altura de las letras es de 5,2 cm. Se descubrió en las excavaciones de la villa (UE 2226; inv. 9991). Se conserva en el Museo de Cerámica de Paterna, donde lo describió y fotografió R. Cebrián.

$$
\text { [M(odii)] XVIII [s(extarii) ---] }
$$

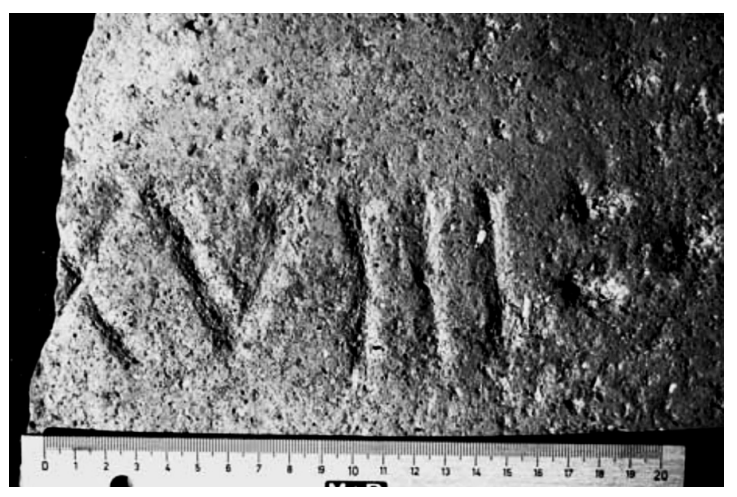

Fig. 8. Grafito de capacidad en el fragmento de dolium $\mathrm{n}^{\circ} 5$.
Aunque en ocasiones se ha supuesto que la capacidad de los dolia era mayor que la indicada por los numerales exteriores (Invernizzi et al. 1999), el estudio de Brenni (1985: 299) prueba que los grafitos mencionan la capacidad en modios cuando van precedidos de la abreviación M (Brenni 1985: 197) ${ }^{14}$.

\section{GRAFITOS CERÁMICOS (figs. 9 a 11)}

Vaso de terra sigillata Hispanica de la forma Dragendorff 37a, partido en 14 fragmentos y hoy reconstruido. Su diámetro es de $17 \mathrm{~cm}$. Está decorado con dos frisos de círculos concéntricos y al exterior, por debajo del labio y en tres puntos diferentes de su circunferencia, presenta otros tantos grafitos post cocción grabados con un punzón o punta seca. Se descubrió en las excavaciones de la villa (UE 4226; inv. 27906). Agradecemos a los responsables de la excavación las fotografías y los datos de la pieza, que pudimos ver en el Museo de Cerámica de Paterna en diciembre de 2012.

a)

Felix

emit

b)

K Iuli

c)

EN

El último de los grafitos (c) parece incompleto y el segundo (b) parece un genitivo que indicaría la posesión de la pieza. La letra inicial de este segunto texto es, con seguridad,

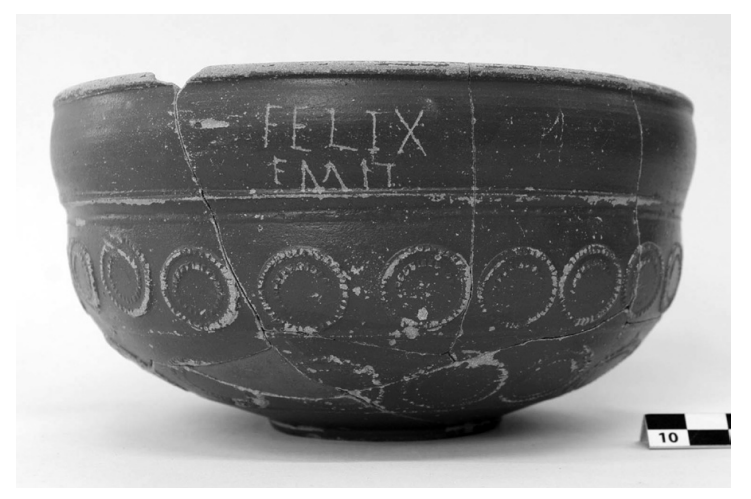

Fig. 9. Grafito a) en el exterior de la pieza $n^{\circ} 6$. 


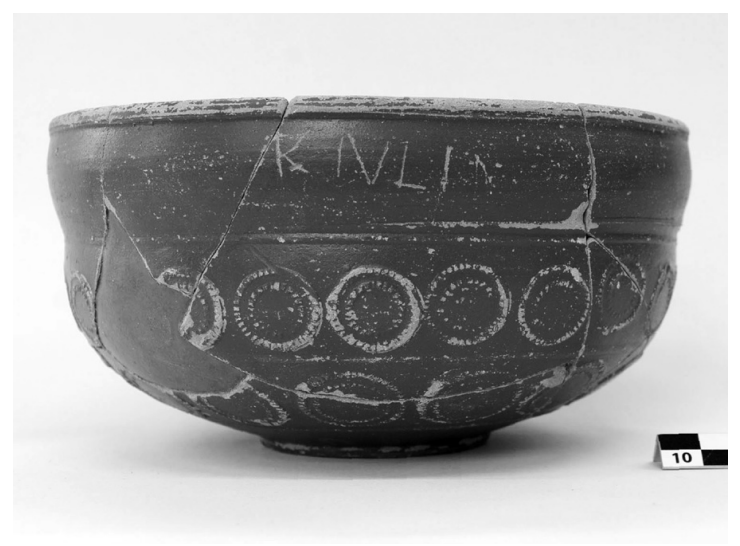

Fig. 10. Grafito b) en el exterior de la pieza ${ }^{\circ} 6$.

K y no R, pues la letra no está cerrada por arriba, con lo que en esa letra habría que entender $\langle C>(a i)$.

El grafito más interesante es el que presentamos aquí como (a), aunque no hay ningún dato sobre el orden en que fueron escritos. En este texto dice claramente Felix emit, es decir, Félix lo compró. Desconocemos el uso del verbo emo,-ere con este sentido en recipientes cerámicos, pues difiere netamente de la expresión eme me bono tuo que se lee en un grafito sobre un dolium de Alvarelhos (Santo Tirso, Porto. AE 1992, 989). Aunque el empleo del verbo emo es corriente en la literatura antigua (Plauto, Terencio, Cicerón, Horacio, Suetonio, etc.), en el ámbito epigráfico se usa habitualmente para aludir a la compra de espacios funerarios ${ }^{15} \mathrm{o}$ de urnas de incineración ${ }^{16}$, la mayor parte de las veces en la propia ciudad de Roma y sólo algunas veces en otros puntos de Italia. En la península Ibérica el uso del verbo emo,-ere esta documentado también en la sentencia del Bronce de Botorrita del año 87 a.C. (CIL I 2951a; $A E$ 1979, 377) donde expresamente se menciona el agrum quem Sallvienses ab Sosinestaneis emerunt.

La forma del vaso sugiere una datación a finales del siglo I o en el siglo II.

\section{VALENCIA. CALLE PALOMAR No 8}

\section{INSCRIPCIÓN FUNERARIA (fig. 12)}

Fragmento de una placa de de caliza de Buixcarró (Saetabis), con la cara frontal pulida y la posterior sólo alisada. Sus dimensiones son $(14,2)$ x $(7,5)$ x $3 \mathrm{~cm}$. El texto está incompleto y sólo conserva parte de tres

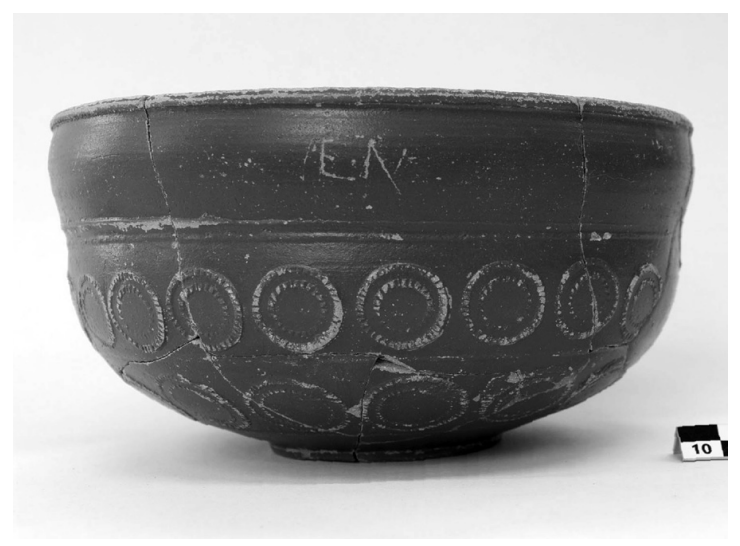

Fig. 11. Grafito c) en el exterior de la pieza $n^{\circ} 6$.

líneas, con letra capital cursiva de buena factura. Se conservan las líneas de pautado que ciñen el segundo renglón, grabadas durante el proceso de ordinatio del texto. La altura de las letras es de 3,2 cm, mientras que los espacios interlineares miden $3 \mathrm{~cm}$. No presenta interpunciones. Fue descubierto en un solar de la calle Palomar $\mathrm{n}^{\circ} 8$, de Valencia durante las excavaciones llevadas a

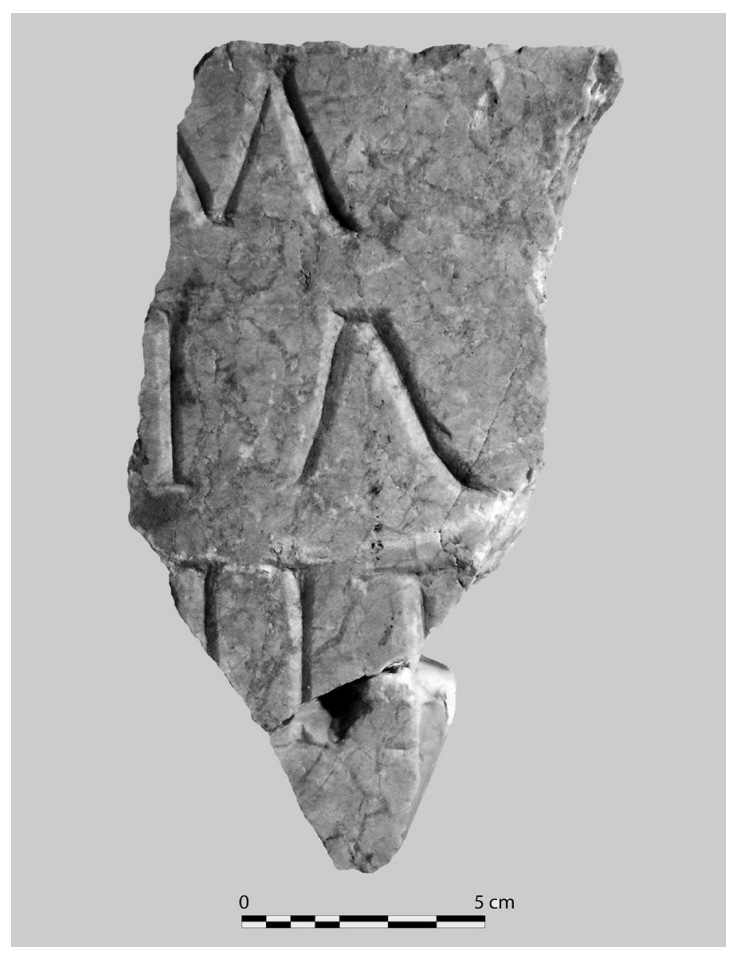

Fig. 12. Fragmento epigráfico de la calle Palomar, en Valencia. 
cabo por D. Guillermo Pascual, a quien agradecemos los datos y la imagen del fragmento, así como la autorización para darlo a conocer.

[D(is)] M(anibus) [s(acrum)?]

$[---] I A[---]$

$[---]+I+[---]$

$-----?$

1: desconocemos si la fórmula dedicatoria incluía la voz sacrum, pues queda un espacio libre a la derecha de la M que tanto puede indicar el final de un renglón como ser el espacio libre antes de la tercera letra. - 2: debería faltar alguna letra después de la A, pues la separación de los caracteres es aquí muy grande y la ordinatio sugiere que el texto continuaba aún tras la fractura. - 3: el trazo central es claramente una I; por delante hay un asta vertical que tanto podría ser otra I como parte de una N; por la derecha se conserva la cabecera de una B, D ó R, por lo que cualquier propuesta de lectura es insegura.

La fórmula inicial y el tipo de letra permiten fechar la inscripción en el s. II.

\section{VALENCIA. CALLE LA PURÍSIMA No 3}

\section{INSCRIPCIÓN FUNERARIA (fig. 13)}

Fragmento de una crusta moldurada de caliza marmórea de Buixcarró (Saetabis), rota a izquierda y derecha y por abajo, que conserva una parte original del borde superior. La superficie frontal está pulida. Sus dimensiones

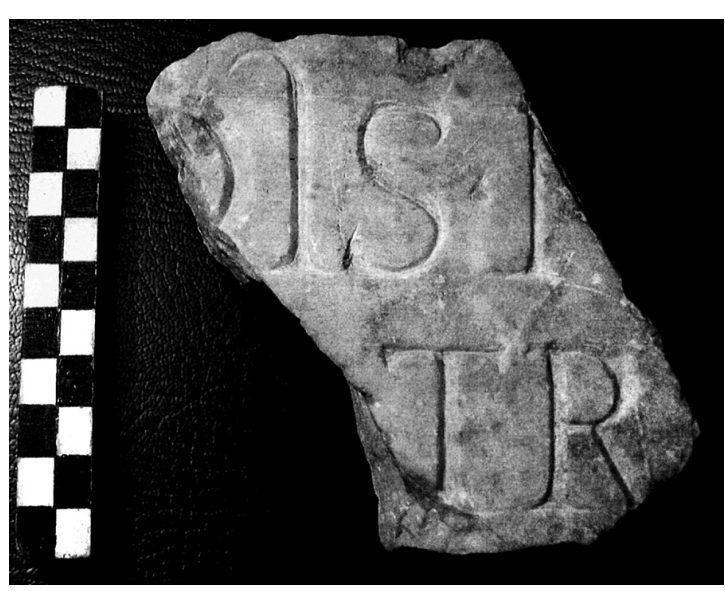

Fig. 13. Fragmento epigráfico de la calle La Purísima, en Valencia. son 11,5 x $12 \mathrm{~cm}$. En el primer renglón conserva una interpunción triangular aparentemente triangular. Fue descubierto en diciembre del año 2003 en un solar de la calle La Purísima $n^{\circ} 3$, de Valencia, durante las excavaciones dirigidas por D. Ignacio Hortelano (UE 1615; inv. $\mathrm{n}^{\circ}$ PUR3/1615/048), a quien agradecemos los datos de la pieza, la fotografía y el permiso para publicarla. El fragmento de epígrafe apareció dentro del relleno de amortización, datado en el siglo $\mathrm{X}$ de una fosa vertedero de época califal (UE 1616), en compañía de abundante material residual de época romana imperial.

\section{Dis $\cdot$ M[an(ibus] \\ [---]TR[---]}

En la primera línea, la forma de abreviar la invocación a los Dioses Manes sugiere que no se grabó al final la voz sacrum. Lo conservado en la segunda línea debe ser parte del nombre del difunto.

El tipo de letra, especialmente la forma de la I, sugiere una datación en la primera mitad del s. II.

\section{RIBA-ROJA DE TÚRIA. VILLA ROMANA DEL POU DE LA SARGUETA}

La villa romana del Pou de la Sargueta, a unos $13 \mathrm{Km}$ al E de la ciudad de Valencia, estuvo enclavada en la antigüedad dentro del territorium de la Valentia romana. Se trata de un caso excepcional de combinación de pars urbana y pars rustica, que fue excavada casi en su integridad por D. Ignacio Hortelano y D. Daniel Sanfeliu y de la que conocemos la planta completa, cuya planimetría y datos de excavación han sido publicados recientemente (Hortelano 2007: 115-126). De este lugar ya se habían dado a conocer previamente algunos epígrafes en forma de grafitos cerámicos, a los que vienen a sumarse ahora un fragmento de inscripción funeraria $\left(n^{\circ} 9\right)$, un grafito con medida de dolium ( $\left.\mathrm{n}^{\circ} 10\right)$ y un pequeño ponderal que está en curso de publicación.

\section{INSCRIPCIÓN FUNERARIA (fig. 14)}

Fragmento de una placa de mármol claro con vetas grisáceas y muchas concreciones en superficie, roto por todos los lados y con la cara anterior pulida. Sus dimensiones son $11,1 \mathrm{x} 12,5 \mathrm{~cm}$. Las letras miden $2,5 \mathrm{~cm}$ de altura y no 


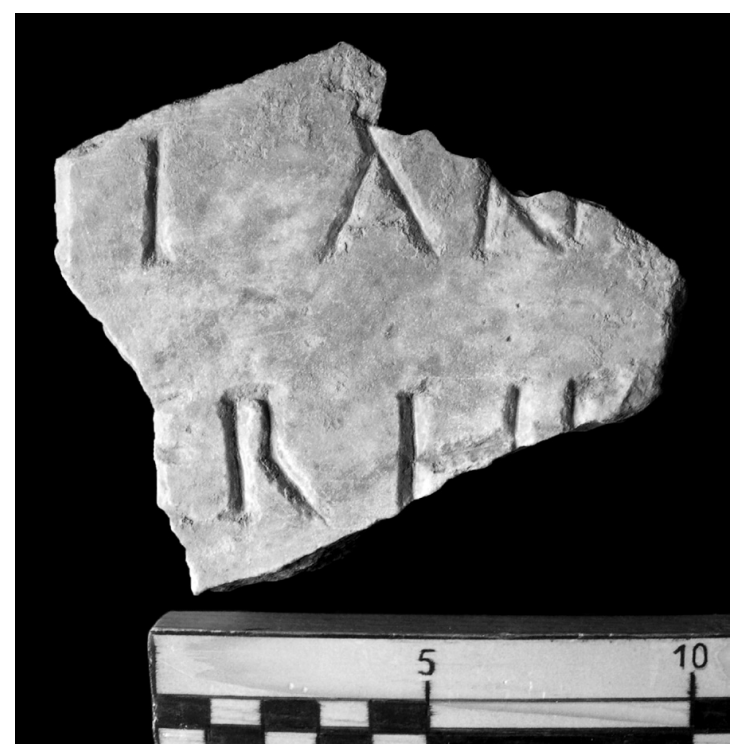

Fig. 14. Fragmento funerario del Pou de la Sargueta, de Riba-Roja de Tùria.

hay huellas de interpunciones. Fue descubierto durante las excavaciones de abril del año 2006 al E de la pars rustica, en un nivel alterado y afectado por labores agrícolas actuales (UE 1008; inv. ${ }^{\circ}$ PSII/1008/pto002/001). Agradecemos a los directores de la excavación los datos, la fotografía y la autorización para publicarlo.

$-----?$

$[---]+I$ (vacat) AN[---]

[---]er (vacat) $\mathrm{H}+[---]$

$-----?$

Las roturas de la pieza impiden incluso saber a qué parte de la inscripción original pertenece. Al comienzo del primer renglón, en la misma fractura, se conserva el surco de un asta vertical que pudo pertenecer a una I, una $\mathrm{M}$ o una $\mathrm{N}$; detrás de ese trazo, a una cierta distancia, se ven las letras AN seguidas de un espacio aparentemente libre, por lo que podría tratarse de la abreviatura de an(norum) pero no hay seguridad de ello. En el segundo renglón, en la línea de fractura, hay una E que ha perdido el asta vertical y de la que sólo se conservan los extremos de los tres trazos horizontales; sigue a continuación una $\mathrm{R}$ con el ojo sin cerrar, un espacio libre y una $\mathrm{H}$ con el trazo central algo inclinado; detrás de la $\mathrm{H}$ hay un asta vertical que parece tener un trazo superior, por lo que podría pertenecer a una $\mathrm{E}$, aunque ese trazo

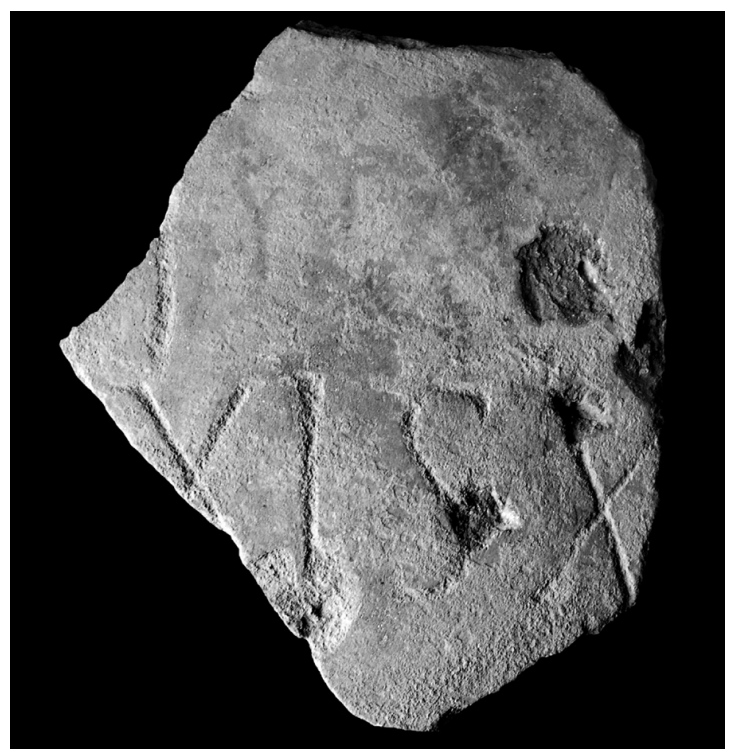

Fig. 15. Grafito de capacidad en el fragmento de dolium n. ${ }^{\circ} 10$.

podría ser sólo parte de la fractura; no se puede descartar el inicio de una fórmula funeraria pero no hay seguridad de ello.

El tipo de letra, ligeramente cursiva, sugiere una datación en la segunda mitad del s. II o primera mitad del III.

\section{INSCRIPCIÓN DE CAPACIDAD (fig. 15).}

Fragmento de pared de un dolium en pasta ocre que presenta al exterior un grafito post cocción con la indicación de su capacidad. Fue descubierto durante las excavaciones de abril del año 2007 en un vertedero situado al exterior (parte oriental) del Edificio A (establos) (UE 1687; inv. $\mathrm{n}^{\circ}$ PSIII/1687/pto071/002). El contexto cerámico permite datar el estrato de hallazgo en el s. IV o a inicios del V. Agradecemos a los directores de la excavación los datos, la fotografía y la autorización para publicarlo.

[M(odii) ---]XI s(extarii) X[---]

Habitualmente, la $\mathrm{S}$ con la que se abrevia la palabra sextarii va situada -como aquí- entre numerales y aparece cruzada por una barra oblicua; pese a carecer de ella, la posición de la letra no deja lugar a duda sobre la lectura. Sobre la capacidad de este tipo de recipientes, véase lo dicho anteriormente para el fragmento $\mathrm{n}^{\circ} 5$. 


\author{
Juan Manuel Abascal Palazón \\ Universidad de Alicante \\ juan.abascal@ua.es \\ Rosario Cebrián FernándeZ \\ Universidad Complutense de Madrid
} marcebri@ucm.es

\section{NOTAS}

1. Este trabajo se ha realizado en el marco del proyecto de investigación HAR2012-32881 de la Secretaría de Estado de Investigación, Desarrollo e Innovación del Gobierno de España. Agradecemos a los arqueólogos D. Carlos Verdasco y D. Pablo Sañudo, de la empresa Estudios Patrimoniales, la autorización para el estudio y publicación de estas piezas, así como los datos que sobre el descubrimiento nos han proporcionado. También queremos agradecer al Director del Museo de Paterna, D Ernesto Manzanedo, las facilidades para llevar a cabo la inspección directa de las mismas.

2. Primera publicación en Corell 1985: 212-214 n. ${ }^{\circ} 3$ con foto; $I d$. 1988: 203 con foto (de ahí, AE 1987, 703c; HEp 2, 1990, 705); Clauss en CIL II ${ }^{2} / 14$, 116; Corell 1997: 253-254 n. 137 (HEp 7 , 1997, 1015) e $I d .2009: 259-260 \mathrm{n}^{\circ}{ }^{\circ} 176$, en ambos casos con foto e importantes discrepancias en la lectura del texto.

3. López Melero y Stylow 1995: 219-253, que aluden a la costumbre generalizada, sobre todo, en Asia Menor de elevar documento público de las disposiciones sepulcrales y las correspondientes conminaciones de multa en el archivo de una comunidad. Para la publicación del documento bastaba con añadirlo a la inscripción de la tumba (ibid., 238 nota 74).

4. El último de los testimonios conocidos es la nueva placa de la lex Ursonensis (Caballos 2006; cf. Caballos 2008: 439-452); una recopilación de los testimonios béticos puede verse en González Fernández 2008. No faltan las evidencias en otros puntos de la península Ibérica, con algunas piezas de excepcional calidad, como $A E 1989,456$, procedente de Andelos.

5. Piénsese en algunos textos votivos, incluso en Hispania: $C I L \mathrm{II}$ 5299 (Malpartida de Cáceres, Cáceres), CIL II 2523 (Viana do Bolo, Orense), CIL II 2819 (Uxama, Soria), AE 1962, 75 (Évora), 1955, 253 (Italica, Sevilla)

6. Mallon y Marín 1951: 131, n. ${ }^{\circ} 272$ (= HAE 722), de Alange, Badajoz. Cf. Álvarez Martínez 1973: 491 n.o 11.

7. Ebovina podría ser una forma derivada de Ebovius/-a, un nombre no documentado hasta ahora; $c f$. sin embargo el nombre céltico Bovius/- $a$ en un buen número de inscripciones del $\mathrm{O}$ de Hispania (EE 9, 121; EE 8, 300; AE 1994, 887; AE 1991, 949 $A E$ 1990, 493; HAE 1146; etc.) Vide también Bovianus (AE 1993, 880 de Dornes, Ferreira do Zezere, Santarem).

8. Práctica de la que no faltan los ejemplos: $c f . A E$ 1987, 602 (Picote, Miranda do Douro, Bragança); HEp 5, 1995, 169 (Ahigal, Cáceres); $A E$ 1996, 854 (Cárquere, Resende, Viseu); $A E$ 1985, 519 (S. João da Fresta, Mangualde, Viseu); $C I L \mathrm{II}^{2} / 5,1149$ (Écija, Sevilla); CIL II ${ }^{2} / 7,287$ (Córdoba); HEp 5, 1995, 245 (Santa Ana, Cáceres).; etc. No faltan los ejemplos en tierras valencias ( $c f . H E p$ 5, 1995, 812 de Oliva, Valencia). La cronología de estos testimonios va del siglo I a finales del II e incluso comienzos del III.

9. Ejemplos del adverbio hic abreviado como hi(c) se conocen en Hispania sobre diversos epígrafes: hi(c) s(itus) e(st) (CIL II 5719, de León), hi(c) s(itus) e(st) AE 1997, 875 (Valmartino, León), hi(c) s(itus) e(st) (HEp 1, 1989, 380, de Aleje, León), hi(c) s(itus) e(st) (AE 1987, 708, de Gandía, Valencia), etc. Tampoco faltan de la abreviación del participio situs/-a como si(tus) o si(ta): hic si(tus) est (AE 1967, 217 de Campolugar, Cáceres), [h(ic)] si(ta) e[st] (CIL II 3075, Toledo), etc.

10. López 1916: 376 (de quien depende Alföldy 1987: 250-251, n. 1 que no pudo ver la inscripción y corrigió la fórmula en la manera en que se publicó en $A E$ 1987, 671. Posteriormente, el propio Alföldy vio la placa y corrigió la lectura en sus apuntes inéditos. Uno de nosotros (J. M. A.) pudo ver la inscripción en compañía de Alfonso Caballero Klink y M. P. González-Conde el 22 de febrero de 2013 y comprobar la lectura.

11. La imagen ha sido dada a conocer por Gimeno 2010: 255-256.

12. Lupulus: CIL II 5621, CIL II 2535 y AE 2007, 839 (un texto cristiano). Lupula: CIL II 4160, AE 1966, 192, AE 1984, 572 y HEp 16, 186. Vide Abascal 1994: 404-406.

13. En Saguntum, el matrimonio de libertos Cn. Baebius Eros Chilonianus y Oppia Montana construyeron en vida su monumento funerario, de 20 pies de lado, que no pasó al heredero (CIL $\mathrm{II}^{2} / 14,407$ ); otro matrimonio de libertos de la gens Popillia hicieron también mención expresa de esta cláusula jurídica $(C I L$ $\left.\mathrm{II}^{2} / 14,645\right)$, al igual que el matrimonio formado por M. Rubrius M. l. Firmamus y Baebia Cn. L. Quieta (CIL II $\left.{ }^{2} / 14,601\right)$.

14. Agradecemos estas observaciones y la referencia bibliográfica a D. Vicent Escrivà.

15. CIL VI 2716 (Roma: Ennia L.f. Tertia emit sibi et L. Mon[t] anio filio suo ...); CIL VI 3636 (Roma: L. Val. Fronto... emit hoc praedium et in hac area fecit...); AE 1969/70, 42 (Roma: D. M. Gavinia Flora et Gavinius Heliodor. emerunt sibi et suis et libertis libertabusque posterisque eorum); AE 1980, 150 (Roma: Arphocras... se vivo locum emit sibi et Corintho...); AE 2001, 533 (Roma: Rufinianus se vivus locum sibi emit); etc.

16. CIL VI 6002 (Roma: A. Camerius C.f. Ani. frater locumemit ollam unam); CIL VI 4554 (Roma: M. Cornelius Alexander Felix libert. et Apollonius libert. emerunt ollam unam); etc.

\section{BIBLIOGRAFÍA}

ABASCAL, J. M. (1994): Los nombres personales en las inscripciones latinas de Hispania. Murcia.

ALFÖLDY, G. (1987): Epigraphica Hispanica 10. Inschriften von Unfreien aus Toledo und Umgebung, Zeitschrift für Papyrologie und Epigraphik 67, 249-262.

ALFÖLDY, G.; HALFMANN, H. (1973): El edetano M. Cornelius Nigrinus Curiatius Maternus. General de Domitiano y rival de Trajano, Serie de Trabajos Varios del SIP 44, Valencia.

ALVAREZ MARTÍNEZ, J. M. (1973): Alange y sus termas romanas, Revista de Estudios Extremeños 29 (3), 431-494.

BELTRÁN LLORIS, F. (1999): Inscripciones sobre bronce: ¿un rasgo característico de la cultura epigráfica de las ciudades hispanas?, XI Congresso Internazionale di Epigrafia Greca e Latina, Roma, 18-24 settembre 1997. Atti, Roma 1999, vol. II, 21-37.

BRENNI, G. M.A., (1985): The Dolia and the Sea-Borne Commerce of Imperial Rome, Tesis Doctoral. Texas A\&M University.

CABALLOS, A. (2006): El nuevo bronce de Osuna y la política colonizadora romana, Sevilla. 
CABALLOS, A. (2008): ¿Típicamente romano? Publicación de documentos en tablas de bronce, Gerión 26 (1), 439-452.

CIL II = HÜBNER, E. (1869/1892): Corpus Inscriptionum Latinarum. Volumen secundum. Inscriptiones Hispaniae Latinae, Berlin 1869. - Corpus Inscriptionum Latinarum. Voluminis secundi supplementum. Inscriptionum Hispaniae Latinarum supplementum, Berlin 1892.

CIL II $2 / 5$ = STYLOW, A. U. et al. (1998): Corpus Inscriptionum Latinorum. Editio altera. Pars 5. Conventus Astigitanus. Berlin - New York.

CIL II $2 / 7$ = STYLOW, A. U. et al. (1995): Corpus Inscriptionum Latinorum. Editio altera. Pars 7. Conventus Cordubensis. Berlin - New York.

CIL II ${ }^{2} / 14$ = ALFÖLDY, G. et al. (1995): Corpus Inscriptionum Latinorum. Editio altera. Pars 14. Conventus Tarraconensis, Pars meridionalis. Berlin - New York.

CORELL, J. (1985): Noves inscripcions romanes del País Valencià, Afers 2, 203-226.

CORELL, J. (1988): L'epigrafia romana al Pais Valencià (1982-1986), Epigrafía (M. Mayer e I. Rodà), Fonaments 7, 196-218.

CORELL, J. (1997): Inscripcions romanes de Valentia i el seu territori. Valencia.

CORELL, J. (2009): Inscripcions romanes del País Valencià, V: Valentia i el seu territori. Amb la col-laboració de X. Gómez Font, Valencia.

DE HOZ, J. (1999): Los metales inscritos en el mundo griego y periférico y los documentos celtibéricos en bronce, Pueblos, lenguas y escrituras en la Hispania prerromana. Actas del VII Coloquio sobre lenguas y culturas paleohispánicas.
Zaragoza, 12 a 15 de marzo de 1997 (F. Villar y F. Beltrán Lloris, eds.), Salamanca, 433-470.

DESSAU, H. (1892/1979): Inscriptiones Latinae selectae I-III, Berlin 1892/1916 (citamos por la edición anastática Chicago 1979).

GIMENO, H. (2010): Fotos vetustas, lecturas nuevas: dos estelas romanas del Castillo de Alarcón, Doctrina a magistro discipulis tradita. Estudios en Homenaje al profesor doctor don Luis García Iglesias (A. J. Domínguez Monedero y G. Mora, eds.), Madrid, 251-258.

GONZÁLEZ FERNÁNDEZ, J. (2008): Epigrafía jurídica de la Bética, Roma.

HORTELANO, I. (2007): El pou de La Sargueta (Riba-Roja de Turia, Valencia), una villa en el territorio de Valentia, Lucentum 26, 115-126.

INVERNIZZI, R. et al. (1999): Scavo di un dolium a Broni (Pavia), Archeología, Uomo, territorio, 18, 53-61.

KAJANTO, I. (1965): The Latin Cognomina (Comment. Human. Litt. 6, 2), Helsinki.

LÓPEZ, V.F. (1916): Nuevo descubrimiento, Revista de Toledo 2, 376.

LÓPEZ MELERO, R.; STYLOW, A. U. (1995): Una pena sepulcral en favor de la res publica Aiungitanorum, Espacio, Tiempo y Forma, Serie II, H. ${ }^{a}$ Antigua, vol. 8, 219-253.

MALLON, J.; MARÍN, T. (1951): Las inscripciones publicadas por el Marqués de Monsalud 1897-1908. Estudio crítico. Madrid.

STYLOW, A. U. (1995): Los inicios de la epigrafía romana en la Bética. El ejemplo de la epigrafía funeraria, Roma y el nacimiento de la cultura epigráfica en Occidente, Zaragoza, 219-238. 